

\title{
Le coût du logement selon sa localisation Jean Cavailhès
}

\section{To cite this version:}

Jean Cavailhès. Le coût du logement selon sa localisation. Informations sociales, 2009, 155, pp.38-46. hal-02654882

\section{HAL Id: hal-02654882 \\ https://hal.inrae.fr/hal-02654882}

Submitted on 29 May 2020

HAL is a multi-disciplinary open access archive for the deposit and dissemination of scientific research documents, whether they are published or not. The documents may come from teaching and research institutions in France or abroad, or from public or private research centers.
L'archive ouverte pluridisciplinaire HAL, est destinée au dépôt et à la diffusion de documents scientifiques de niveau recherche, publiés ou non, émanant des établissements d'enseignement et de recherche français ou étrangers, des laboratoires publics ou privés. 


\title{
CA I R N
}

chercher : repérer : avancer

Cet article est disponible en ligne à l'adresse :

http://www.cairn.info/article.php?ID REVUE=INSO\&ID NUMPUBLIE=INSO 155\&ID ARTICLE=INSO 1550038

\section{Le coût du logement selon sa localisation}

\author{
par J ean CAVAILHÈS
}

\section{| Caisse nationale des Allocations familiales | Informations sociales}

\section{$2009 / 5-N^{\circ} 155$}

ISSN 0046-9459 | pages 38 à 46 conditions générales d'utilisation du site ou, le cas échéant, des conditions générales de la licence souscrite par votre établissement. Toute autre reproduction ou représentation, en tout ou partie, sous quelque forme et de quelque manière que ce soit, est interdite sauf accord préalable et écrit de l'éditeur, en dehors des cas prévus par la législation en vigueur en France. Il est précisé que son stockage dans une base de données est également interdit. 


\section{Le coût du logement selon sa localisation}

Jean Cavailhès - économiste

Les prix d'achat et les loyers des logements, qui pèsent de plus en plus lourd dans le budget des ménages, varient fortement selon les départements et à l'intérieur de ceux-ci. De quelle manière la position du logement dans la hiérarchie urbaine influe-t-elle sur le niveau de la dépense qui lui est consacrée? Comment l'économie explique-t-elle les variations observées?

Le logement représente le poste de dépense le plus important des ménages et il est en forte augmentation : les dépenses courantes de logement, nettes des aides personnelles, sont passées de $17 \%$ du revenu disponible brut des ménages en 1984 à près de $23 \%$ en 2006 (Briant et Rougerie, 2008). Si bien que les accidents de paiement se multiplient : 565000 ménages accédants et 1,8 million de locataires ont eu des difficultés à payer leur remboursement d'emprunt immobilier ou leur loyer en 2006 (Briant et Rougerie, 2008).

Le coût du logement varie fortement dans l'espace. Au troisième trimestre 2008, le prix moyen départemental de maisons individuelles de quatre pièces dépasse 250000 euros dans vingt-deux départements (Île-de-France exclue) pour la plupart situés sur les façades maritimes, dans le quart sud-est du pays, et dans les départements où se trouvent de grandes métropoles (Rhône, Haute-Garonne, Bas-Rhin, etc.). Ce prix est inférieur à 150000 euros dans dix départements situés le long de la «diagonale du vide», qui traverse la France du plateau lorrain au Massif central. Au total, il varie entre 868000 euros (Alpes-Maritimes) et 120000 euros (Creuse).

Le prix moyen d'un appartement dépasse 2500 euros le $\mathrm{m}^{2}$ dans dix-huit départements (dont tous ceux d'Île-de-France) et il est inférieur à 1300 euros le $\mathrm{m}^{2}$ dans quatorze départements. Il va de 6630 euros le $\mathrm{m}^{2}$ (Paris) à 1070 euros le $\mathrm{m}^{2}$ (Meuse), soit une amplitude de 1 à plus de 6. 
Cependant, il faut tenir compte des variations intradépartementales : le prix dans les grandes villes (préfectures, etc.) est plus élevé que dans les petites villes et les communes rurales. Il s'agit ici d'expliquer ces variations spatiales par l'économie urbaine. À cet effet, la localisation des logements est analysée selon la typologie de l'Institut national de la statistique et des études économiques (Insee), qui distingue 354 aires urbaines, chacune étant constituée par un pôle urbain, ensemble d'une ville-centre et de sa banlieue qui offre plus de 5000 emplois, et par une ceinture périurbaine, qui envoie plus de $40 \%$ de sa population active travailler dans le pôle urbain. C'est ainsi que l'aire urbaine de Paris s'étend jusqu'à près de $100 \mathrm{~km}$ de la capitale et compte plus de 10 millions d'habitants, alors que les plus petites aires urbaines dépassent à peine le seuil de 5000 emplois et ont un rayon de quelques kilomètres. Les ceintures périurbaines comptent près de 10000 communes et s'étendent sur le tiers du territoire.

Nous ne traitons pas des aspects temporels, qu'il s'agisse des cycles (rapporté au revenu disponible des ménages, le prix des logements a connu des périodes de hausse : 1975 - 1980, 1986 - 1991 et surtout 2000 - 2008, entrecoupées de périodes de baisse : 1969 - 1975, 1980 - 1985, 1992 - 1996 - voir Friggit, 2009) ou des évolutions récentes. En prenant la fin de l'année 2000 comme référence (indice 100), les prix étaient à un indice voisin de 115 en 1992, puis ils ont baissé d'environ $30 \%$ en 1998 et ont ensuite été multipliés par presque trois entre 1998 et 2008, qui marque le haut du cycle immobilier. Un examen plus précis montre que les évolutions qui viennent d'être rappelées sont comparables dans les régions françaises. Les prix ont augmenté un peu moins vite à Paris ou en petite couronne francilienne qu'en ProvenceCôte d'Azur, un peu plus vite en Rhône-Alpes que pour la moyenne de la province, par exemple, mais les mouvements vont tous dans le même sens. Leur ampleur est comparable : pour une base 100 en 2000, l'indice au quatrième trimestre 2008 est compris entre 211 (zones rurales et petites villes de province) et 246,5 (Provence-Alpes-Côte d'Azur).

C'est pourquoi nous raisonnons le plus souvent ici en affectant un indice égal à 100 à une localisation spatiale donnée, quelle que soit l'année d'observation, pour examiner les variations de cet indice dans l'espace. Certaines données utilisées dans la suite de cet article sont des moyennes obtenues à partir des enquêtes Logement de 1984 à 2002 de l'Insee. Il y a eu, depuis, une évolution en niveau (les prix ont augmenté, les surfaces également, etc.) mais la répartition spatiale, qui est la question étudiée ici, n'a pas beaucoup varié depuis 2002.

Ces enquêtes Logement permettent d'appliquer la méthode économétrique des « prix hédonistes ", grâce à laquelle on peut estimer le prix de chacun des attributs élémentaires d'un logement (surface habitable, confort, localisation et environnement, etc.) dont le détail n'est pas directement donné sur le marché, puisqu'on n’observe que le prix ou le loyer global du logement. 


\section{Graphique - Indices du prix des appartements selon la zone géographique}



Source : indices Notaires-Insee, d'après Friggit (2009).

On obtient ainsi des prix qui respectent la condition «toutes choses égales par ailleurs ». Ces enquêtes, représentatives du parc de logements, permettent de décrire les variations des loyers et des prix d'achat des logements selon deux variables fondamentales en économie urbaine : la taille des villes et la distance aux centres d'emploi. En effet, le coût du logement s'explique, en premier lieu, par un arbitrage entre le coût des migrations alternantes (vers les emplois des centres urbains) et le coût foncier (on peut avoir des logements plus grands lorsqu'on s'éloigne des villes).

\section{L'arbitrage entre coût foncier et coût de déplacement}

Les économistes expliquent la localisation résidentielle des ménages par l'arbitrage entre le coût de leurs migrations alternantes domicile/travail et le coût de leur logement. D'un côté, un ménage est incité à se rapprocher du centre-ville, qui concentre l'essentiel des emplois et des services, pour réduire son coût de déplacement ; d'un autre côté, ce gain entraîne un renchérissement du coût du logement, ce qui réduit la taille de celui-ci pour un budget donné. Lorsque le gain d'un déplacement plus court est compensé par le surcoût du logement engendré par ce rapprochement, il n'y a pas de raison de déménager, puisque gains et pertes s'équilibrent. L'équilibre urbain est ainsi atteint. 
La diminution des valeurs foncières lorsqu'on s'éloigne du centre-ville joue un rôle crucial dans cette analyse. Or, le coût automobile connaît une baisse tendancielle. Selon les indices des prix de l'Insee et déduction faite de l'inflation, une voiture s'achetait, en 1975, moitié plus chère qu'aujourd'hui (à qualité constante) et le prix relatif de l'essence a lui aussi baissé (malgré les chocs pétroliers). De plus, la vitesse de déplacement a augmenté, en particulier avec les améliorations du réseau routier, et les voitures sont devenues moins gourmandes en énergie, du fait du progrès technique. Au total, le coût généralisé de transport, défini comme la somme du coût monétaire direct (voiture, essence, entretien) et du coût du temps de transport (valorisé sur la base du salaire horaire), a baissé au cours du dernier demi-siècle. Cette baisse a favorisé l'extension des villes loin des centres urbains. C'est ce qui a amené l'Insee à définir des aires urbaines, qui sont des zones souvent assez vastes sous l'influence d'une ville-centre ou d'un pôle urbain.

\section{Le coût du logement dans l'aire uribaine de Paris}

Prenons le cas de l'aire urbaine de Paris, suffisamment grande pour que les enquêtes Logement de l'Insee soient représentatives. En posant au niveau 100 l'indice moyen du loyer des appartements par mètre carré de surface habitable dans Paris intra muros, l'indice tombe à 65 en banlieue (pour fixer les idées : entre 5 et $15 \mathrm{~km}$ de Notre-Dame), puis il est égal à environ 55 entre 15 et $50 \mathrm{~km}$ de la capitale, et à 47 en périphérie lointaine, soit plus de deux fois moins cher qu'à Paris. Cette réduction du loyer par mètre carré avec la distance permet de se loger dans des appartements plus vastes : leur superficie est de $53 \mathrm{~m}^{2}$ dans Paris, de $58 \mathrm{~m}^{2}$ à $5-15 \mathrm{~km}$ et de $65 \mathrm{~m}^{2}$ audelà de $15 \mathrm{~km}$. Cependant, la taille moyenne des ménages augmente lorsqu'on s'éloigne

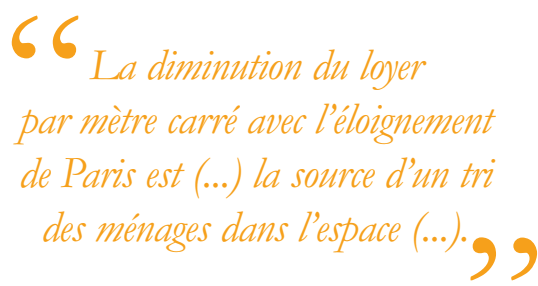
de Paris : un ménage compte, en moyenne, 1,65 unité de consommation (UC) dans Paris et jusqu'à 2,14 UC dans la couronne périurbaine. Il en résulte que la surface habitable disponible par UC est d'environ 33 à $36 \mathrm{~m}^{2}$ quel que soit l'éloignement, sauf en périphérie très lointaine, où elle monte à 42 $\mathrm{m}^{2}$ par UC.

La diminution du loyer par mètre carré avec l'éloignement de Paris est donc la source d'un tri des ménages dans l'espace: ceux de petite taille habitent de petits appartements dans Paris et les grands ménages recherchent des appartements plus grands en périphérie, où le loyer est plus faible, ce qui permet de maintenir une surface habitable par UC à peu près constante dans toute la région.

Pour ce qui concerne les maisons individuelles en propriété, le prix unitaire diminue de 1,2\% par kilomètre d'éloignement de Paris, ce qui est un rythme plus rapide que celui des appartements en location (- 0,7\% par kilomètre). 
Pour ce segment du parc de logements, la surface habitable par UC dépend peu de la distance et elle est plus importante que pour les locataires d'appartement : en moyenne, 45,6 $\mathrm{m}^{2}$ par UC contre 35,2.

C'est surtout la surface du jardin qui augmente lorsqu'on s'éloigne de Paris : en ne prenant pas Paris en compte (il y a trop peu d'observations de maisons individuelles avec jardin dans les enquêtes Logement), elle est d'environ 350 à $450 \mathrm{~m}^{2}$ jusqu'à une vingtaine de kilomètres de Notre-Dame, puis elle augmente régulièrement et dépasse $1100 \mathrm{~m}^{2}$ vers la frontière de l'aire urbaine.

\section{Le prix du logement dans la hiérarchie urbaine}

Le coût d'un logement ne dépend pas seulement de la distance au centre urbain, mais aussi de la population de celui-ci. En effet, un ménage habitant en périphérie d'une grande ville ou d'une grande aire urbaine supporte un coût de déplacement vers le centre urbain plus élevé que dans une unité moins peuplée. Comme le coût de déplacement est nul dans les deux cas pour un ménage qui habite au centre, la compensation coût de déplacement/coût foncier se traduit par un coût du logement supérieur au centre d'une grande agglomération. C'est ce que permet de constater le tableau.

\section{Tableau - Caractéristiques des maisons individuelles en propriété et des appartements en location selon la taille de la commune centre des aires urbaines}

\begin{tabular}{|c|c|c|c|c|c|}
\hline Taille des aires urbaines & $\mathrm{Pa}$ & iris & $\begin{array}{r}\text { Com } \\
\text { ce } \\
\text { plu } \\
100000\end{array}$ & $\begin{array}{l}\text { mune } \\
\text { ntre } \\
\text { Is de } \\
\text { habitants) }\end{array}$ & $\begin{array}{c}\text { Commune } \\
\text { centre } \\
\text { (moins de } \\
100 \text { 000 habitants) }\end{array}$ \\
\hline Distance & $\begin{array}{c}\text { Paris intra } \\
\text { muros }\end{array}$ & $\begin{array}{c}\text { Banlieue } \\
(5 \text { à } \\
7,5 \mathrm{~km})\end{array}$ & Centre & $\begin{array}{c}\text { Proche } \\
\text { banlieue } \\
(<5 \mathrm{~km})\end{array}$ & Centre \\
\hline $\begin{array}{l}\text { Maisons individuelles en propriété } \\
\text { Prix d'achat par } \mathrm{m}^{2} \\
\text { Surface habitable } \\
\text { Surface habitable par UC } \\
\text { Surface terrain }\end{array}$ & $\begin{array}{l}- \\
- \\
- \\
-\end{array}$ & $\begin{array}{c}100 \\
110 \\
47 \\
377\end{array}$ & $\begin{array}{l}- \\
- \\
- \\
-\end{array}$ & $\begin{array}{c}46 \\
116 \\
51 \\
649\end{array}$ & $\begin{array}{c}41 \\
111 \\
49 \\
759\end{array}$ \\
\hline $\begin{array}{l}\text { Appartements en location } \\
=\text { Loyer par } \mathrm{m}^{2} \\
\text { Surface habitable } \\
\text { Surface habitable par UC }\end{array}$ & $\begin{array}{c}100 \\
52 \\
34\end{array}$ & $\begin{array}{l}61 \\
57 \\
33\end{array}$ & $\begin{array}{l}55 \\
61 \\
39\end{array}$ & $\begin{array}{l}45 \\
67 \\
40\end{array}$ & $\begin{array}{l}43 \\
63 \\
41\end{array}$ \\
\hline
\end{tabular}

Source : enquêtes Logement de l'Insee. Indice 100 : loyer par mètre carré des appartements dans Paris ou prix d'achat par mètre carré des maisons à $5-7,5 \mathrm{~km}$ de Paris (le nombre de maisons dans Paris est trop faible dans ces enquêtes pour être pris comme référence). 
Pour les maisons individuelles, par rapport à la référence 100 en banlieue parisienne, le prix tombe à l'indice 46 en proche banlieue des grandes aires urbaines de province et à 41 au centre des plus petites. Cependant, cette division du prix par plus de deux n'entraîne qu'un accroissement négligeable de la surface habitable de la maison. C'est principalement le jardin qui profite d'une rente foncière moins onéreuse : sa taille passe de moins de $400 \mathrm{~m}^{2}$ en banlieue parisienne à $650 \mathrm{~m}^{2}$ en proche banlieue des grandes agglomérations de province et à $760 \mathrm{~m}^{2}$ au centre des plus petites.

En ce qui concerne le loyer par mètre carré des appartements, en prenant la valeur 100 dans Paris (soit, en 2008, 23 euros le $\mathrm{m}^{2}$ par mois), l'indice est de 55 dans les grandes agglomérations de province (par exemple, en 2008, 11 euros le $\mathrm{m}^{2}$ par mois à Lyon) et de 43 dans les petites (par exemple, en 2008, 7 euros le $\mathrm{m}^{2}$ par mois à Rodez). C'est la surface habitable qui profite de cette baisse du loyer unitaire : elle passe de $52 \mathrm{~m}^{2}$ dans Paris $\left(34 \mathrm{~m}^{2}\right.$ par $\mathrm{UC})$ à $61 \mathrm{~m}^{2}$ dans les grandes villes de province $\left(39 \mathrm{~m}^{2}\right.$ par UC) et à $63 \mathrm{~m}^{2}$ dans les petites aires urbaines $\left(41 \mathrm{~m}^{2}\right.$ par UC). Notons que le loyer du secteur social est nettement plus faible que celui du secteur libre : en moyenne, il est de $30 \%$ inférieur.

Le taux d'effort brut des accédants à la propriété est inférieur à celui des locataires et, en 2002, il diminuait avec la taille des aires urbaines : il atteignait $30 \%$ du revenu dans l'aire urbaine de Paris et il était de 25,8\% dans les petites aires urbaines de province. Le taux d'effort brut des locataires du secteur libre était, en 2002, supérieur à $45 \%$ du revenu dans les grandes aires urbaines de province et inférieur à $40 \%$ dans les petites (42\% dans l'aire de Paris). La hiérarchie était la même en déduisant les aides au logement pour obtenir un taux d'effort net: celui-ci était de $36 \%$ dans les grandes aires urbaines (Paris et province) et de $30 \%$ dans les petites. Ces taux d'effort étaient sensiblement inférieurs dans le secteur HLM (entre 34 et $38 \%$ pour le taux brut et entre 22 et $26 \%$ pour le taux net), sans que l'on observe les mêmes variations que pour le secteur libre selon la taille des agglomérations.

\section{Le prix des caractéristiques propres du logement}

Les résultats qui suivent sont obtenus par la méthode économétrique des prix hédonistes, dont nous avons dit qu'elle respecte la condition «toutes choses égales par ailleurs ». Ils indiquent ainsi l'effet spécifique sur le loyer d'une caractéristique donnée d'un logement (sa surface habitable, sa localisation, etc.), toutes les autres caractéristiques étant inchangées. Le loyer d'un logement dépend, en premier lieu, de la surface habitable. Chaque mètre carré supplémentaire fait augmenter le loyer de 1,2\%. Les salles d'eau sont l'élément de confort essentiel : le loyer augmente de $15 \%$ pour une salle de bain ou un WC supplémentaire.

Le bâtiment où est situé le logement influe également sur le loyer. Il s'agit, tout d'abord, de la date de construction : les habitations neuves ont un loyer 
supérieur de moitié à celles de la période 1949 - 1974, qui inclut la période de construction de masse des années 1960, où l'on a construit beaucoup de logements de basse qualité. Le loyer est également élevé pour les immeubles construits avant la Seconde Guerre mondiale, tout particulièrement à Paris et dans les plus grandes villes de province. Ensuite, la taille de ce bâtiment

La distance fait diminuer le loyer de $1 \%$ environ par kilomètre d'éloignement (à vol d'oiseau)

par rapport au centre

de l'aire urbaine. intervient dans l'aire urbaine de Paris et dans les plus grandes aires urbaines de province, où le loyer diminue d'environ $19 \%$ dans les immeubles comptant plus de cinquante logements. L'état de l'immeuble est aussi pris en compte dans ces deux types d'aires urbaines, le loyer diminuant de 8 à

$10 \%$ lorsqu'un élément important (ascenseur, façade, etc.) n'est pas en bon état. Enfin, les dégradations subies par l'immeuble du fait d'actes de vandalisme déprécient le loyer de $13 \%$ dans l'aire urbaine de Paris, qui est la seule où cet attribut ait une valeur significativement négative. La taille de l'immeuble, son état et les dégradations qu'il peut avoir subies sont des attributs liés aux "grands ensembles", qui se rencontrent plus souvent en banlieue de Paris ou des grandes métropoles, ce qui explique que leur influence sur le loyer ne soit significative que dans ces types urbains. En revanche, contrairement à ce que l'on aurait pu attendre, les agressions, vols ou cambriolages n'ont pas d'effet négatif, quelle que soit la taille de l'aire urbaine.

\section{Le prix de la localisation}

La distance fait diminuer le loyer de $1 \%$ environ par kilomètre d'éloignement (à vol d'oiseau) par rapport au centre de l'aire urbaine. Il semble que ce prix de la distance soit plus faible dans les grandes aires urbaines que dans les plus petites.

La richesse de la commune, évaluée à partir du revenu imposable des foyers fiscaux, a une grande influence sur le loyer. Cela provient de ce que les écoles et services publics sont de meilleure qualité que la moyenne dans les communes habitées par des ménages riches, qui sont donc prêts à payer un loyer plus élevé, et de ce que les ménages aisés bénéficient du voisinage de pairs, ce qui les conduit également à louer plus cher que là où ils seraient mêlés à des ménages plus pauvres. En partant de situations moyennes (c'est-à-dire de la médiane de la distribution des communes selon le revenu net imposable des foyers fiscaux), le loyer d'un logement est de $40 \%$ inférieur dans une commune moyennement pauvre (à la moitié de cette médiane). À l'inverse, en montant de la médiane vers une commune moyennement riche (située à $75 \%$ dans la hiérarchie de la richesse des communes), le loyer progresse de $6 \%$, puis à nouveau de près de $14 \%$ en passant à une commune plus riche ( $90 \%$ de la hiérarchie). C'est l'une des variables les plus influentes en dehors des caractéristiques propres du logement. 
Les différences de loyer entre communes riches et communes pauvres sont particulièrement importantes en région parisienne.

Certaines autres variables d'aménités ou de nuisances liées à l'environnement du logement ont des effets sur le loyer plus faibles que les précédentes, ou statistiquement non significatifs. Il en est ainsi des bruits nocturnes, de la pollution de l'air, de l'exposition du logement ou d'une vue dégagée. Un quartier dégradé - parce que la voirie est insuffisante, que les rues sont sales ou les terrains vagues nombreux, etc. - peut faire baisser les loyers d'environ $1 \%$. La décote atteint environ $3 \%$ pour les quartiers mal desservis (manque de commerces, de transports en commun, d'équipements socioculturels ou sportifs). La banlieue parisienne n'obéit pas ici à la même règle, semblant au contraire privilégier l'éloignement de ces services.

Les résultats globaux ainsi résumés varient selon le type d'espace : par exemple, le prix du mètre carré de plancher représente $2,1 \%$ du loyer annuel dans l'aire urbaine de Paris et il oscille entre 1,1 et 1,5\% du loyer dans les aires urbaines de province. La pression foncière explique que les jardins des maisons individuelles aient, dans les pôles des grandes aires urbaines, une valeur supérieure à ceux des petites, sans parler de la banlieue parisienne, où ils atteignent des sommets. Parce qu'il connote une bonne qualité de l'immeuble dans une ville où l'on est avide d'air, un balcon à Paris vaut quatre fois plus qu'en province.

Les grands ensembles déprécient les loyers en comparaison des petits immeubles, bien plus dans l'aire urbaine parisienne (- 18,1 \% du loyer pour les logements dans des immeubles de plus de cinquante logements) et dans les grandes métropoles de province (- $19 \%$ du loyer) que dans les plus petites agglomérations. De même, les immeubles dégradés entraînent des décotes du loyer de $13 \%$ dans la région parisienne et de 1 à $4 \%$ ailleurs. En région parisienne, dans les communes où le revenu moyen des ménages est faible (situé dans le quart inférieur), le loyer est déprécié de 77,5\%. En province, c'est une localisation dans les communes où le revenu moyen est élevé (situé dans les $10 \%$ supérieurs) qui renchérit, parfois de beaucoup, les loyers. Rappelons que l'ensemble de ces résultats sont des estimations obtenues par un modèle statistique.

\section{Éléments de conclusion}

Le logement est le poste de dépense le plus important des ménages français. Les locataires et les accédants à la propriété y consacrent plus du quart de leur revenu, parfois plus de la moitié. Ce coût est en augmentation depuis un quart de siècle. Or, le prix d'un logement varie fortement dans l'espace. Premièrement, il est deux fois plus élevé dans les centres urbains que dans leur périphérie (qui s’étend parfois à plusieurs dizaines de kilomètres et à une centaine de kilomètres pour l'aire urbaine de Paris) ; deuxièmement, il est deux fois plus élevé dans les grandes villes (Paris en premier lieu) que dans les 
petits centres urbains, sans parler de l'espace rural ; l'hétérogénéité du parc de logements est source d'une troisième cause de variations (taille des immeubles, état et dégradations du bâti, etc.) ; et l'hétérogénéité sociale de l'espace, d'une quatrième (communes riches et communes pauvres, etc.). selon leurs caractéristiques propres (budget, nombre d'enfants, goûts, etc.). Cela se traduit par un «tri spatial» : les ménages qui se ressemblent ont spontanément tendance à choisir les mêmes localisations. On a pu dire que le Code civil était une machine à hacher la terre (du fait des divisions ; on peut formuler, de même, que le marché immobilier est une machine à hacher la société, regroupant ici les pauvres, là la classe moyenne, ici les familles nombreuses, ailleurs les célibataires, les jeunes, les retraités, etc.

À ce tri «spontané » opéré par le marché s'ajoutent souvent des politiques publiques discriminatoires. En particulier, les politiques foncières locales, par les caractéristiques des plans locaux d'urbanisme (taille des lots résidentiels, coefficients d'occupation des sols, etc.) et la fiscalité foncière, accentuent ce tri et opèrent une véritable ségrégation spatiale des groupes sociaux. De nombreux travaux montrent que les inégalités spatiales/sociales se sont accrues depuis un quart de siècle (voir, entre autres, Cavailhès et Selod, 2003). Afin de promouvoir une mixité sociale, il faut donc mettre en œuvre des politiques spatiales du logement et des politiques urbaines suffisamment puissantes pour contrebalancer le tri du marché immobilier et la ségrégation des politiques publiques locales.

\section{Bibliographie}

ఐ Briant P. et Rougerie C., 2008, " Les logements sont plus confortables qu'il y a vingt ans et pèsent davantage sur le revenu des ménages ", France, portrait social, édition 2008, Insee, p. 103-116.

ఐ Cavailhès J., 2005, « Le prix des attributs du logement ", Économie et statistique, $\mathrm{n}^{\text {os }} 381-382$, p. 91-123.

" Cavailhès J. et Selod H., 2003, "Ségrégation sociale et périurbanisation ", Inra sciences sociales, $\mathrm{n}^{\circ} 1-2 / 03,4 \mathrm{p}$.

- Friggit J., 2009, http://www.adef.org/statistiques/index.htm 